\title{
Age characteristics of the working memory
}

\author{
Elena Nikolaeva $^{1,{ }^{*}, \text { Elvira } \text { Dunaevskaya }}{ }^{1}$, Svetlana Burkova $^{1}$, Svetlana Nikiforova $^{1}$, and \\ Vera Merenkova $^{2}$ \\ ${ }^{1}$ Herzen State Pedagogical University, Saint Petersburg, nab reki Moki 48, 191186, Russian \\ Federation \\ ${ }^{2}$ Yelets State Ivan Bunin University, Yelets, ul. Kommunarov, 28, 399770, Russian Federation
}

\begin{abstract}
This paper raises the question of the relationship between two mechanisms of working memory - Retrieval-Induced Forgetting (RIF) and Retrieval-Based Learning (RBL) in ontogenesis. Working memory is an element of executive functions, the effectiveness of which predetermines the success of learning, which determines the importance of studying the mechanisms of its implementation. RIF is a deterioration in the reproduction of subsequent stimuli as a result of reproduction of previous stimuli that are similar in some parameter. RBL is the reverse process, in which each subsequent reproduction in the working memory leads to better memorization of information when the memory is repeatedly tested. Comparison of works representing specific ages of the subjects does not allow us to imagine the complete change in the interaction of the two mechanisms with age. This is what became the task of this study. An original computerized technique was used (Razumnikova et al., 2016) which had been designed to memorize visual objects presented on a computer screen. The technique included three series, during which the same set of simple objects were presented, but the order of the presentation varied from series to series. The study involved 201 children: 17 children who were 3-4 years old, 90 children who were 5-7 years old, 47 children 10-11 who were years old and 47 children who were 12-14 years old. It is shown that RIF processes are mostly formed in children 3-4 years old. The effectiveness of RBL increases with age and reaches its greatest values by adolescence.
\end{abstract}

\section{Introduction}

The modern understanding of the regulation of behavior is associated with two different states: stereotyped behavior, which automatically repeats a certain sequence of actions, and behavior change, requiring additional attention and implementation mechanisms. Stereotypical behavior is that the same actions are repeated, and the same regulatory sequences govern them. This is what people do all the time, not noticing that they are doing it: they go to the subway, put a card through the validator, enter the carriage, etc. But at some point, these actions must be stopped in view of the changed situation (for example, a pandemic and the transition to a position of self-isolation). Habitual automated actions no

\footnotetext{
${ }^{*}$ Corresponding author: elenlou@yandex.ru
} 
longer correspond to the changed tasks, and therefore new functions must be activated that put the body into manual control mode. These functions are called executive functions.

Executive functions are called the totality of descending mental processes necessary for concentration of attention and control of behavior when automatic, instinctive, intuitive behavior becomes ineffective or impossible. The constituent elements of executive functions are working memory, inhibitory control and cognitive flexibility [1]. Inhibition control ensures the cessation of previous ineffective actions at the moment, working memory provides memorization of the final result and intermediate stages, and cognitive flexibility helps to change behavior when the situation changes.

The relevance of studying the effectiveness of working memory lies in the fact that it is the basis of learning processes, since it is strongly associated with attention [2], the formation of new motor and sensory skills [3], provides language acquisition [4], reading processes [5] arithmetic operations [6]. That is why a decrease in the functioning of working memory leads to certain difficulties in learning, in particular, a connection with language problems, dyslexia and difficulties in reading mastering, dyscalculia [7] has been proven. Moreover, the deterioration of WM is reflected in the very process of learning at school: from a decrease in the level of memorization and execution of instructions to the impossibility of planning and solving educational problems [8].

Working memory has a limited volume (the number of elements that are simultaneously used for analysis), since the brain has a limited cognitive resource. It develops most actively in the first 10 years in relation to other periods of human life [9] and reaches the level of development in adults by about 14 years [10].

The function of the working memory is to link the elements of the actual problem that stood earlier with those that will be implemented later, as well as control the sequence of the solution, keeping the intermediate results of all stages [11]. At the same time, all these intermediate elements can change, transform, which makes it possible to realize the goal in changing conditions. That is why the preservation of intermediate results until the end of the task ensures the success of its implementation.

Several theoretical models of WM functioning are currently being discussed. One of the first models, the componential model, is based on the assumption that WM is a special type of memory and includes a number of constituent parts, which are separate compartments for storing various types of information. For 40 years this model has been constantly modernized [12] and has shown significant efficiency.

However, according to Cowan [12], WM is not a separate type of memory, but is an activated part of long-term memory (LTM). There is currently no reliable data to give preference to one point of view or another, since the forecasts of these two models are sometimes very close.

The most discussed mechanisms operating in working memory are retrieval-induced forgetting (RIF) and retrieval-based learning (RBL) [13]. RIF is a deterioration in the reproduction of subsequent stimuli as a result of reproduction of previous stimuli that are similar in some parameter.

There is no uniform understanding of the RIF mechanism. One of the mechanisms for the deterioration of reproduction is considered to be proactive interference, that is, the imposition of memories of previous stimuli on the memorization of new information and weakening the formation of a new memory trace [14]. Theories explaining the effect of interference suggest that the search signal activates several memories simultaneously, and the competition of memory traces prevents accurate memory. That is, forgetting seems to be a consequence of the associative structure of memory and the proximity of the reproduced information.

Anderson and Bjork [15] suggested that forgetting is not a consequence of the competition of memory traces, but is due to a special active inhibitory process acting during the search for an answer. To facilitate memorization of the target stimulus, the non-target at 
the moment is suppressed, and it is this mechanism that impairs the possibility of subsequent reproduction.

Norman, Newman, and Detre [16] proposed a theoretical approach that combines these two mechanisms and explains forgetting by the action of both interference and inhibition. Moreover, in this paradigm, inhibition occurs when competing units are activated. However, both inhibition and interference are of particular importance in relation to the theories that have come together in this new view.

It should be emphasized that proactive interference is considered an element of inhibitory control, and therefore a greater RIF effect is observed with effective inhibitory processes [17].

It is known that inhibitory functions that gradually appear in ontogenesis are detected very early and are formed quite late. Their activity largely depends on the maturation of the prefrontal cortex. This area of the brain is one of the last to appear, and therefore its maturation is the longest in relation to the structures found in evolution much earlier [18].

RBL is the opposite of the RIF process, which means that each subsequent reproduction in the working memory leads to better memorization of information after repeated testing. It is known that RBL accompanies the memorization of stimulus material of different quality and volume [19].

The final result of reproduction is determined by the interaction of two processes: RIF and RBL.

However, it has been shown that RIF correlates to a greater extent with the final result of memorization, and an increase in RIF was found in individuals with better memory when performing an arithmetic task, while the RBL effect did not correlate with the volume of memorized material.

Nevertheless, there is an opinion that RBL is a fundamental mechanism in learning and development of intellectual abilities. Both RIF and RBL depend on the activity of the prefrontal cortex. According to the neuroanatomical model, prefrontal inhibitory modulation of memory trace reproduction includes three pathways: switching attention to processing other stimuli, inhibition of an individually specific pattern of memory representation, and generalized inhibition of the hippocampus and, accordingly, reactivation of reproduction [20]. Such a variety of functional systems of inhibition, apparently, determines the widespread variability of the mechanisms of reproduction of the memory trace.

Variability can also be determined by age characteristics, since both the mechanisms of memorizing information and the mechanisms of inhibitory control and interference change with age. Their change may be due to the very process of maturation of the prefrontal cortex, on which these processes rely, as well as myelination processes in other areas of the brain, which accelerate the conduction of information along the axon and the connectivity of brain structures. However, the comparison of works representing specific ages of the subjects does not allow us to present a complete picture of changes in the interaction of the two mechanisms with age. This is what became the task of this study: to study the age-related characteristics of the relationship between retrieval-induced forgetting and retrieval-based learning in working memory.

\section{Materials and methods}

To clarify the age-related patterns of the interaction between RIF and RBL in working memory, an experimental model of visual memory testing with the effect of both RIF and RBL was used.

An original computerized technique was used [21], designed to memorize visual objects presented on a computer screen. The technique included three series, during which the same set of simple objects (30 units) was presented, but the order of presentation varied from series 
to series. The objects were objects familiar to children of all ages (leaves, butterflies, twigs, bugs, etc.)

The subject initially saw three objects, from which he had to select any and click on it with the mouse cursor. Subsequently, new objects were gradually added, and the subject was asked to mark with the mouse cursor the object that had not been marked earlier. If the subject made a mistake and pressed the object that he had already chosen earlier, then the series ended, and a new presentation of the same stimuli began with a different order of their appearance. Another mistake led to the end of this series and the beginning of a new one. The number of correctly reproduced elements in each series was counted and the ratio of the number of reproduced elements in different series was estimated.

The time of object selection was not regulated. For statistical data processing, the IBM SPSS Ver.22 software package was used.

The study involved 201 children: 17 children that were 3.0-4.5 years old, 90 children that were 5-7 years old, 47 children that were 10-11 years old and 47 children that were 12-14 years old. The choice of age is due to data on the development of working memory in ontogenesis. Informed consent was obtained from the parents of the children for the participation of children in the study. All children attended one or another educational institution (kindergarten, preschool children or secondary school, older children).

\section{Results}

The analysis was started by comparing the volumes of reproduction by children of all groups. Table 1 shows the results of three reproductions by subjects of each group.

Table 1. The number of reproduced elements in three series and the age of the subjects in the groups (mean and standard deviation).

\begin{tabular}{|l|l|l|l|l|}
\hline $\begin{array}{l}\text { Groups } \\
\text { (age in years) }\end{array}$ & age & Recall 1 & Recall 2 & Recall 3 \\
\hline $3.0-4.5$ & $4.0 \pm 0.9$ & $10.0 \pm 4.8$ & $5.6 \pm 4.7^{*}$ & $3.1 \pm 1.4^{* *}$ \\
\hline $5.0-7.0$ & $6.4 \pm 0.8$ & $15.6 \pm 7.3$ & $10.1 \pm 6.0$ & $10.4 \pm 5.2$ \\
\hline $10.0-11.0$ & $10.6 \pm 0.4$ & $13.9 \pm 6.8$ & $10.8 \pm 6.4$ & $10.8 \pm 6.0$ \\
\hline $12.0-14.0$ & $13.4 \pm 1.5$ & $12.0 \pm 7.5$ & $9.0 \pm 5.0$ & $8.1 \pm 5.0$ \\
\hline
\end{tabular}

Note: $^{*}$ significant differences in reproduction were noted between the group of children that were 3.0-4.5 years old and older children with a significance level of $p \leq 0.05$ and $^{\text {*** }} \mathrm{p} \leq 0.01$ (Student's test).

Table 1 shows that the first reproduction does not differ significantly in the subjects of different groups, included because of the rather large scatter of indicators, although there is a tendency of less reproduction of elements by the subjects of the youngest group. However, the second and third reproduction in children of the youngest group is significantly worse than in older children. As noted earlier, in all groups, on average, RIF processes prevailed over RBL processes.

Table 2 shows the ratio of RIF and RBL processes in children in different groups.

The table shows that RIF processes prevail in children of the younger group, and only two children have both the learning effect and the forgetting effect (the second reproduction is better than the first, and the third is significantly worse than the first two).

The RIF effect in the remaining groups gradually decreases and has a minimum value in children 10-11 years old, while the RBL effect increases, and half of adolescents that were 12-14 years old had both effects.

Of the 17 children of the first group, 3.0-4.5 years old, $88.2 \%$ had the RIF effect, $11.8 \%$ had RBL. For those who had an improvement in memory, it occurred in the second attempt, and memory impairment was noted in the third attempt. 
Among 90 children that were 5-6 years old, a variety of situations was observed. $74.4 \%$ had only the RIF effect, $45.6 \%$ had only RBL, and $22.2 \%$ had both effects. In the latter case, the first reproduction was low (3-5 elements), and therefore the changes were within one or two reproduction units.

Table 2. RIF and RBL ratio (\%) in different age groups.

\begin{tabular}{|c|c|c|c|c|}
\hline $\begin{array}{c}\text { Groups } \\
\text { (age in years) }\end{array}$ & $\begin{array}{c}\text { Number of } \\
\text { children }\end{array}$ & RIF (\%) & RBL (\%) & $\begin{array}{c}\text { RIF+RBL } \\
(\%)\end{array}$ \\
\hline $3.0-4.5$ & 17 & $100^{*}$ & $11.8^{* *}$ & $11.8^{*}$ \\
\hline $5.0-6.0$ & 90 & 74.4 & 45.6 & 22.2 \\
\hline $10.0-11.0$ & 47 & 59.6 & 63.8 & 29.8 \\
\hline $12.0-14.0$ & 47 & 83.0 & 70.2 & 48.9 \\
\hline
\end{tabular}

Note: designations as in table 1.

RIF effect was noted in $59.6 \%$ of grade 4 pupils out of $47 ; 63.8 \%$ have RBL. $29.8 \%$ have both parameters. In younger adolescents, the RIF effect was found in $83 \%$ of children, RBL - in $70.2 \%$ of children. Consequently, there is an increase in the learning effect with age.

\section{Discussion}

We examined the indicators of working memory in children aged 3-14 years. The choice of age is due to the literature data, which states that this age range corresponds to the intensive formation of working memory. Rapid development begins at the age of 3, and by the age of 14, its volume reaches the adult level [22]. At the same time, according to our data, the ratio of RIF and RBL mechanisms at different stages of ontogeny is not the same.

We found that during the first reproduction, the memory volume in children of different ages does not fundamentally differ due to the large values of the standard deviation in all groups, which may reflect different rates of brain maturation, determined by some other reasons (genetic, prenatal and early postnatal development, nutrition, emotional environment, and stress levels).

However, the volume of memory during the second and third reproduction changes sharply, and the children of the smallest age differ significantly from the children of other groups in the volume of reproduced material (they have a minimal amount). Moreover, in this group, first of all, interference and inhibitory processes are noted and there is practically no learning in the process of reproduction. However, the older the child is, the more likely it is to include RBL. It seems that inhibitory processes are formed earlier in relation to learning processes.

The study of the neuronal structures responsible for working memory in adults indicates a large role in this process of the lateral prefrontal cortex [23]. However, it matures rather late into ontogeny. There is evidence that this may not be earlier than 17 years [24].

A study using the fNIRS technology [25] of visual WM in children 3-4 years old revealed the activation of both the frontal and parietal cortices. Moreover, the activation in the parietal cortex was higher in children of 4 years old compared with children at 3 years old. Consequently, in children of 3 years old, the process of memorizing information requires the inclusion of a larger volume of the brain in the work in comparison with children of 4 years.

A longitudinal study using structural and functional MRI in young children found more structures in relation to older children that are activated during the assessment of working memory [26]. In addition to DL-PFC, activation of a number of subcortical structures were noted; however, the level of activation of different structures with varying degrees of probability allowed predicting the level of WM at a later age. So, at the age of 6, DL-PFCs were activated, but the level of its activation did not allow predicting the effectiveness of 
WM in the future. However, a similar prediction could be made by assessing the activation of the basal ganglia and thalamus. In addition, the fMRI study [46] found that if the ventrolateral PFC was activated in children and adults during WM-related tasks, then in children there was no activation of the right DL-PFC and bilateral activation and superior parietal cortex (it was activated in adults).

A study using fNIRS technology in children 4-10 years old [27] demonstrated that the work of nonspatial WM is ensured by the right inferior frontal gyrus and the orbitofrontal cortex.

Thus, it can be concluded that the frontal and parietal cortex, as well as subcortical structures and connections between them, are the central substrates of WM from infancy and beyond. However, the involvement of the dorsolateral cortex in its work is different, and it is less effective in children.

We have already mentioned that Anderson [28] considers active forgetting to be the mechanism of RIF. Moreover, active forgetting, from his point of view, is not a consequence of unique processes in memory, but is an element of inhibitory control. It is assumed that no special mechanism is needed for forgetting through active inhibition, and mnemonic processes such as storing and encoding information are built into the brake control mechanism. And the lateral prefrontal cortex, especially the right DLPFC, is the basis for the implementation of forgetting,

In young children, working memory and mechanisms of inhibitory control are interdependent, and therefore, with the development of inhibitory processes, the lateral prefrontal cortex is also activated. However, with age, this activation gradually decreases [29].

Inhibitory control is associated with the suppression of unwanted or insignificant stimuli and actions, as well as habitual patterns of behavior, which have now lost their significance. It is formed quite early [1], and some researchers note that already 6 month old children have elements of inhibitory control. At the same time, it is believed that there is a rapid increase in its efficiency during infancy and early childhood. Inhibitory control reaches the adult level already in early adolescence [30].

It has been proven, based on fMRI studies, that the dorsal and ventral-lateral PFC is responsible for the formation of inhibitory control. Moreover, the DL-PFC is also activated in tasks in which WM and inhibitory control are studied simultaneously. However, this activation in tasks in which inhibitory control is assessed decreases with age, and at the same time, the striatum begins to be involved.

In our study, it is important to compare the characteristics of inhibitory processes at different age periods. While fMRI studies can be carried out in adolescence, neuroimaging was not available for young children for a long time. The advent of fNIRS changed the situation. A study of the neuronal substrates of inhibitory processes in children 4-6 years old showed [31] that there is a difference in activation processes in solving problems aimed at assessing inhibitory control in adults and children. In adults, the activation of the right frontal and parietal regions occurs directly in tasks for inhibitory processes, while in children these structures are more active outside of these tasks.

Consequently, it has been proven that rIFC, or more narrowly in some studies, the right ventrolateral PFC, is a key structure in inhibitory processes. However, Aron et al. [32] describes the mechanism of action of this area as follows. He believes that during the formation of inhibitory processes, the rIFC sends signals to the subthalamic nucleus of the basal ganglia, which suppress thalamocortical output and thus prevent the motor response prepared in the cortical structures.

However, this specialization of the rIFC as the main substrate of inhibitory processes is formed rather late in ontogenesis, starting from the preschool period and ending with adolescence. 
At the same time, it is worth emphasizing the ambiguity of the results, which sometimes indicate the important role of both left and bilateral IFC activation [60]. Moreover, some authors reveal a greater activation of the left IFC, and not the right in the process of development [33].

However, what is important in our study is that RIF processes begin to form earlier than $\mathrm{RBL}$, and a pronounced difference is noted between the 3-4 and 5-7 age groups. It can be assumed that it is the different rates of maturation of the processes of memorization and inhibition that lead to different ratios of RIF and RBL in ontogeny, primarily in the age period from 3 to 5 years.

\section{Conclusion}

It was shown that the ratio of the effects of RIF and RBL in WM changes at the age from 34 to 14 years. This change occurs against the background of unchanged average indicators of the volume of the first playback in WM in this age range (with a large individual spread of indicators in each age group). Consequently, the first reproduction is possibly determined by genetic, intrauterine factors or characteristics of the environment in which the child develops and does not depend on those developmental features that determine the formation of RIF and RBL in WM. At the age of 3-4 years, the best formed RIF processes with practically no RBL process. The effectiveness of RBL increases with age and reaches its greatest values by adolescence.

\section{References}

1. A. Diamond, Annual Review of Psychology 64, 35-68 (2013) doi: 10.1146/annurevpsych-113011-143750

2. N. Cowan, N. M. Fristoe, E.M. Elliott, R.P. Brunner, J.S. Saults, Memory and Cognition 34(8) 1754-1768 (2006) https://doi.org/10.3758/BF03195936

3. H.C. Leonard, M. Bernardi, E.L. Hill, L.A. Henry, Developmental Neuropsychology 40(4) 201-215 (2015) https://doi.org/10.1080/87565641.2014.997933

4. C. Weiland, M.C. Barata, H.Yoshikawa, Infant and Child Development 23, 4-21 (2014) https://doi.org/10.1002/icd.1829

5. M.F. Kudo, C.M. Lussier, Y.L. Swanson, Research in Developmental Disabilities 40 51-62 (2015) https://doi.org/10.1016/j.ridd.2015.01.002

6. L. Cragg, S. Richardson, P.J. Hubber, S. Keeble, C. Gilmore, PLOS ONE 12(12), e0188693 (2017) https://doi.org/10.1371/journal.pone.0188693

7. A.J. Jaroslawska, S. E. Gathercole, M.R. Logie, J. Holmes, Memory and Cognition 44 580-589 (2016) https://doi.org/10.3758/s13421-015-0579-2

8. R.G. Alloway, T.P. Alloway, Understanding working memory (2nd ed.), Sage, Los Angeles (2015) DOI: 10.1017/edp.2015.14

9. R.J. Allen, G.J. Hitch, A.D. Baddeley, Visual Cognition 17 (1-2) 83-102 (2009) doi:10.1080/13506280802281386

10. V.S. Merenkova, O.E. El'nikova, E.I. Nikolaeva, Science for Education Today 10(6) 27 39 (2020) DOI: 10.15293/2658-6762.2006.02

11. A.D. Baddeley, Trends in Cognitive Sciences 4(11) 417-423 (2000) https://doi.org/10.1016/S1364-6613(00)01538-2

12. N. Cowan, Progress in Brain Researches. 169, 323-338 (2008) https://doi.org/10.1016/S0079-6123(07)00020-9 
13. C. Cinel, C.C. Mack, G.Ward, Journal of Experimental Psychology: General 147(5) 632- 661 (2018) http://dx.doi.org/10.1037/xge0000441

14. A. Aslan, K.-H.T. Baum, J. Exp. Psychol. Learning, Memory, and Cognition 37(1), 264 269 (2011) http://doi.apa.org/getdoi.cfm?doi=10.1037/a0021324

15. M.C. Anderson, B.J. Levy, In Bjork R.A., Benjamin A.S. (eds). Successful remembering and successful forgetting: A festschrift in honor. Psychology Press Publ. 107-132 (2010)

16. K.A. Norman, E.L. Newman, G. Detre, Psychological Review 114 887-953 (2007) doi:10.1037/0033-295X.114.4.887

17. H.J. Kramer, T.D. Wood, L.K. Hjortsvang, K.H. Lagattuta, Cognitive Development 57, 100975 (2021) https://doi.org/10.1016/j.cogdev.2020.100975

18. B. Pastötter, S. Schicker, J. Niedernhuber, K.-H.T. Bäuml, J. Exp. Psychol. Learn. Mem. Cogn. 37, 287-297 (2011) doi:10.1037/a0021801

19. E.G. Vergunov, E.I. Nikolaeva, N.V. Balioz, S.G. Krivoschekov, Human Physiology 44(3), 320-329 (2018) DOI: 10.1134/S0362119718030143

20. B.E. Depue, Neuroscice and Biobehavioral Review 36(5), 1382-1399 (2012) DOI: 10.1016/j.neubiorev.2012.02.012

21. O.M. Razumnikova, M. Savinykh, R.Suslov, R.O. Petrov, Proc. 11th Intern. Forum on Strategic Technology (IFOST) 256-258 (2016)

22. L. Traverso, P. Viterbori, Ch. Malagoli, M.C. Usai, Cognitive Development 55, 100909 (2020) https://doi.org/10.1016/j.cogdev.2020.100909

23. A. Fiske, K. Holmbo, Developmental Review 52, $42-62$ (2019) https://doi.org/10.1016/j.dr.2019.100866

24. V.L. Efimova, E.I. Nikolaeva, Psychology in Russia: State of the Art1 2(1), 79-88 (2019) DOI: 10.11621/pir.2019.0106

25. A.T. Buss, N.A. Fox, D.A. Boas, J.P. Spencer, Neuroimage 85 (1), 314-325 (2014) doi:10.1016/j.neuroimage.2013.05.034

26. H. Ullman, R. Almeida, T. Klingberg, Journal of Neuroscience 34 (5), 1592-1598 (2014) doi:10.1523/JNEUROSCI.0842-13.2014

27. E.A. Crone, C. Wendelken, S. Donohue, L. van Leijenhorst, S.A. Bunge, Proceedings of the National Academy of Sciences of the United States of America 103 (24), 9315-9320 (2006) doi:10.1073/pnas.0510088103

28. M.C. Anderson, J. C. Hulbert, Annual Review of Psychology 72, 1-36 (2021) https://doi.org/10.1146/annurev-psych-072720-094140

29. Ch. Cheng, Z. Kaldy, E. Blaser, Cognitive Development 55, 100892 (2020) https://doi.org/10.1016/j.cogdev.2020.100892

30. N. Garon, I.M. Smith, S.E. Bryson, Child Neuropsychology 20 (6), 713-736 (2014) doi: 10.1080/09297049.2013.857650

31. F. Verbruggen, G.D. Logan, Trends in Cognitive Sciences 12 (11), 418-424 (2008) doi:10.1016/j.tics.2008.07.005

32. A.R. Aron, T.W. Robbins, R.A. Poldrack, Trends in Cognitive Sciences 18 (4), 177-185 (2014) doi: 10.1016/j.tics.2013.12.003

33. K. Rubia, A.B. Smith, E. Taylor, M. Brammer, Human Brain Mapping 28 (11),11631177 (2007) doi: 10.1002/hbm.20347 